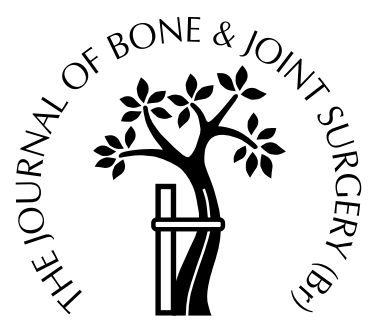

\title{
Changes in the three-dimensional microstructure of human tibial cancellous bone in early osteoarthritis
}

\author{
M. Ding, A. Odgaard, I. Hvid \\ From Aarhus University Hospital, Aarhus, Denmark
}

W e obtained medial and lateral subchondral cancellous bone specimens from ten human postmortem proximal tibiae with early osteoarthritis (OA) and ten normal age- and gender-matched proximal tibiae. The specimens were scanned by micro-CT and the three-dimensional microstructural properties were quantified.

Medial OA cancellous bone was significantly thicker and markedly plate-like, but lower in mechanical properties than normal bone. Similar microstructural changes were also observed for the lateral specimens from OA bone, although there had been no sign of cartilage damage. The increased trabecular thickness and density, but relatively decreased connectivity suggest a mechanism of bone remodelling in early $\mathrm{OA}$ as a process of filling trabecular cavities. This process leads to a progressive change of trabeculae from rod-like to plate-like, the opposite to that of normal ageing. The decreased mechanical properties of subchondral cancellous bone in $\mathrm{OA}$, which are due to deterioration in architecture and density, indicate poor bone quality.

J Bone Joint Surg [Br] 2003;85-B:906-12.

Received 5 July 2002; Accepted after revision 21 March 2003

Osteoarthritis (OA), is a major cause of pain and disability in the elderly. For the past two decades, research into the aetiology of OA has mainly concentrated on the destruction of articular cartilage in which the damage is clearly visible. There are indications, however, that changes occur in all

M. Ding, MD, PhD, Senior Research Scientist

A. Odgaard, MD, DMSci, Consultant Orthopaedic Surgeon

I. Hvid, MD, DMSci, Professor

Orthopaedic Research Laboratory, Aarhus University Hospital ( $\mathrm{KH}$ ), Building 1A, Nørrebrogade 44, DK-8000, Aarhus C, Denmark.

I. Hvid, MD, DMSci, Professor

Department of Orthopaedics, Aalborg University Hospital, Aalborg, Denmark.

Correspondence should be sent to Dr M. Ding.

(C)2003 British Editorial Society of Bone and Joint Surgery doi:10.1302/0301-620X.85B6.12595\$2.00 elements of the joint, and those in subchondral cancellous bone may play a primary role in the pathogenesis of OA. ${ }^{1-4}$

Evidence is accumulating that specific changes in the microstructure of subchondral cancellous bone in OA are consistent with an acceleration of bone turnover. ${ }^{5}$ These structural changes occur through the action of osteoclasts and osteoblasts in selectively removing and adding bone. Structural changes in OA have also been described as differences in trabecular surface and shape compared with normal control groups. ${ }^{6}$ Early increases in horizontal trabecular thickness are followed by later changes in the vertical structures. ${ }^{7}$ Bone mineral density increases in both the axial and peripheral skeleton with progression of $\mathrm{OA}^{8}$ and there may be an abnormal low mineralisation pattern in late-stage OA, even though it is associated with a thickening of the subchondral bone plate. 9

In late-stage OA, the apparent density of cancellous bone is increased, but tissue density is decreased compared with normal controls. ${ }^{5}$ The stiffness of subchondral cancellous bone in OA increases more slowly as apparent density increases than does normal cancellous bone. ${ }^{5}$ Although OA is known to be associated with an increase in cancellous bone density, ${ }^{3}$ little is known about the three-dimensional (3-D) microstructural changes, such as the type of structure, architectural anisotropy and connectivity in cancellous bone with early OA.

The quality of cancellous bone is important in the pathogenesis and development of OA, and has been assessed in late-stage $\mathrm{OA}$ in human hips by the quantification of bone mineral density, microdamage and mechanical properties. ${ }^{10}$ Fazzalari et $\mathrm{al}^{10}$ reported that bone mineral density does not reflect changes in the mechanical properties of cancellous bone as the accumulation of microdamage decreases bone quality in late-stage OA. Until now, research has mainly focused on moderate- or late-stage OA. It remains unclear whether or not the quality of cancellous bone deteriorates in early human OA.

Our aim was to investigate the microstructural changes in subchondral cancellous bone in early human OA. We hypothesised that significant microstructural changes, in addition to changes in density, occur in subchondral cancellous bone in early OA. We assessed which of the microstructural properties could best predict the mechanical properties of cancellous bone in early OA, and whether the 
normal relationship between mechanical and microstructural properties could also be applied to cancellous bone in early OA.

\section{Materials and Methods}

We defined the degree of OA according to the criteria of Mankin et $\mathrm{al}^{11}$ and confirmed this by histological examination. Early OA is characterised by the presence of macroscopically fibrillated cartilage.

The donors, who had all died suddenly from either trauma or acute disease, were all Caucasian. We removed one post-mortem tibia from each donor. Proximal tibiae were obtained from ten donors, seven men and three women with a mean age of 73 years (63 to 81), with early OA. As controls, we removed normal proximal tibiae from ten ageand gender-matched donors, seven men and three women with a mean age of 72 years ( 58 to 85 ).

In OA tibiae, visible degeneration with slight fissures was present on the cartilage surface (superficial zone) of the medial condyle, whereas that of the lateral condyle was intact. Normal tibiae had no macroscopical pathology or history of musculoskeletal diseases, and they had intact cartilage surfaces.

We obtained three cylindrical specimens from each medial and lateral tibial condyle using a $7.5 \mathrm{~mm}$ innerdiameter trephine. These specimens were cut $1 \mathrm{~mm}$ below the subchondral bone plate and then further cut at the distal end to create $7.5-\mathrm{mm}$ long cylindrical specimens. The axis of each specimen corresponded to the longitudinal axis of the tibia. The 120 specimens were stored in plastic tubes at $-20^{\circ} \mathrm{C}$. The early OA specimens were allocated to medial $\mathrm{OA}$ and lateral control groups, and the normal specimens were allocated to normal medial and normal lateral groups, and 30 specimens were examined in each group. The mean Mankin scores for the groups were 4.9 (3 to 7) for the medial OA, 1.7 (1.5 to 3) for the lateral control, 0.8 (0 to 2) for the normal medial, and 0.5 ( 0 to 1 ) for the normal lateral.

We used a high resolution micro-CT system $(\mu \mathrm{CT} 20$; Scanco Medical AG, Zürich, Switzerland) to scan the specimens, resulting in a cubic voxel size of $22 \times 22 \times 22 \mu \mathrm{m}^{3}$. Each 3-D image data set consisted of approximately 350 micro-CT slide images $(512 \times 512$ pixels $)$ with 16 -bit-graylevels. In order to obtain accurate 3-D data sets, micro-CT images were segmented using optimal thresholds so that the bone volume fraction of the datasets corresponded to Archimedes' based volume fraction. ${ }^{12}$ From the segmented accurate 3-D micro-CT data sets, ten microstructural properties were calculated based on true, unbiased and assumption-free 3-D methods. Bone volume fraction (bone voxel per total specimen voxel) was determined. ${ }^{12}$

The structure model index, a measure of predominant shapes in the structure, such as plate-like, rod-like or a combination of plate and rod, ${ }^{13}$ was based on a differential analysis of the triangulated bone surface of a structure. This quantification is especially important for an unknown or changing structure, such as ageing, bone remodelling and disease. The value is 0 for an ideal flat plate structure, and 3 for an ideal cylindrical rod structure. ${ }^{13}$

We determined the 3-D trabecular thickness and bone surface-to-volume ratio (bone surface area per bone volume) for each sample. ${ }^{14}$ For quantification architectural anisotropy, preferential orientation of trabecula, we used a 3-D volume method; star volume distribution. ${ }^{15}$ The degree of anisotropy was defined as the ratio between two eigen values, that is the ratio of the eigen value of the primary direction divided by the eigen value of the tertiary direction. ${ }^{16}$ Unbiased and assumption-free quantification of connectivity (number of trabeculae per volume) was based on a topological approach. ${ }^{17}$ We also determined the trabecular volume, the mean marrow space volume, 3-D trabecular spacing and bone surface density (bone surface area per total volume of specimen). ${ }^{18}$

After scanning, the specimens were tested in compression to failure to determine ultimate stress (strength) and failure energy, ${ }^{19}$ using an 858 Bionix MTS hydraulic material testing machine (MTS Systems Co., Minneapolis, Minnesota) and a $1 \mathrm{kN}$ load cell. The ultimate stress was calculated from the first maximal point with maximal stress, and failure energy was calculated as the area underneath the stress-strain curve between zero strain and ultimate strain.

Statistical analysis. We used SPSS version 10.0 (SPSS Inc, Chicago, Illinois) with the mean value of the three specimens from each condyle being as a variable in all analyses. Two-way analyses of variance were carried out for repeated measurements of the microstructural properties. Comparing $\mathrm{OA}$ and normal groups determined the between-subjects factor and the medial and lateral groups, the within-subjects factor. All the tests were first checked for normality and equal variance. When significant main effects or an interaction between the main effects were found, particular posthoc multiple comparisons between groups were made using the Bonferroni test.

Linear regression analyses assessed associations between mechanical and microstructural properties. The slopes and intercepts of the linear regressions were tested to assess whether there were significant differences in the relationship between the best predictors from the mechanical and microstructural properties in the four groups. For all statistical analyses, exact $\mathrm{p}$ values were given and a $\mathrm{p}$ value $<0.05$ was considered to be significant.

\section{Results}

Examples of the 3-D reconstruction of cancellous bone from micro-CT images for the four groups are shown in Figure 1. Significant changes in the microstructure of cancellous bone in OA can be observed. It has a very heterogeneous architecture. Compared with normal medial control, it is significantly thicker and denser, and unusually plate-like, but is lower in mechanical properties (Fig. 1). 

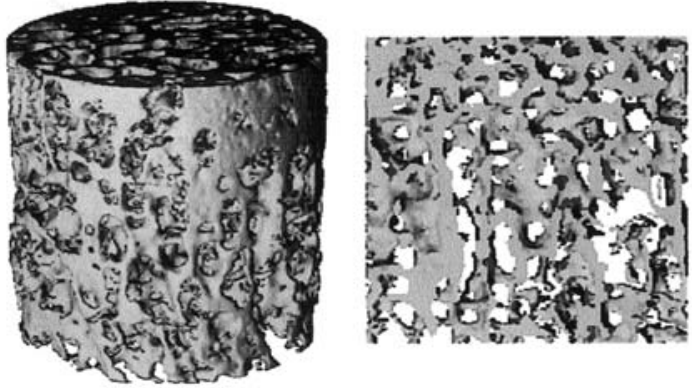

Medial osteoarthritis
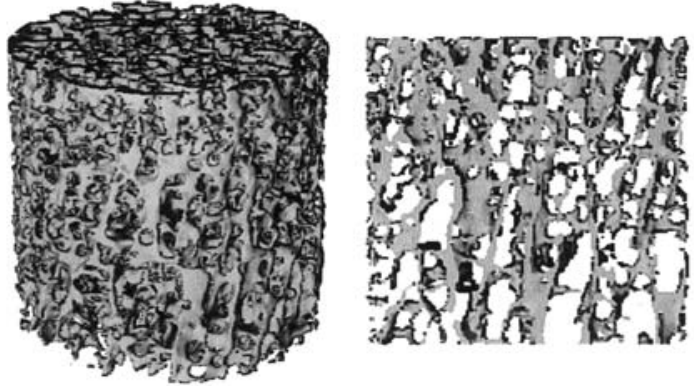

Normal medial

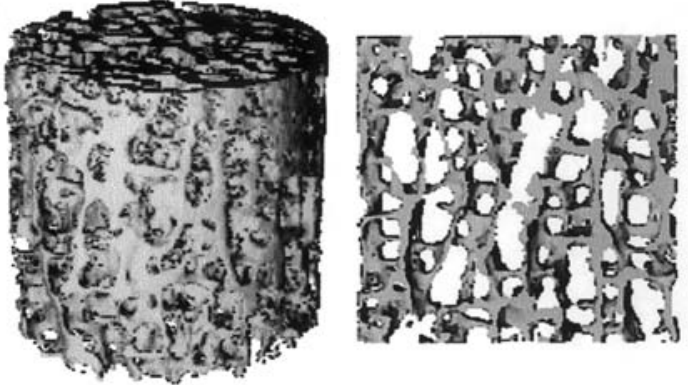

Lateral control
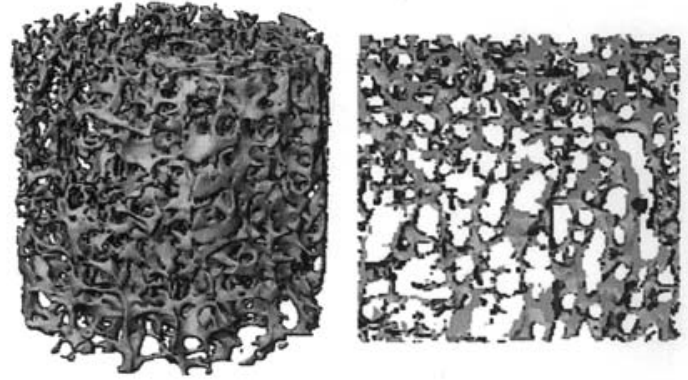

Normal lateral

Fig. 1

3-D reconstructions of cylindrical cancellous bone specimens from micro-CT scans. Comparing the four specimens obtained from cancellous bone of tibiae with early OA, medial specimens (upper left) had the highest bone volume fraction and trabecular thickness, typical plate-like structure and the lowest bone strength and lateral specimens (upper right) had higher bone volume fraction and trabecular thickness, more plate-like structure and lower bone strength. Of the specimens obtained from normal tibiae, medial specimens (lower left) had lower bone volume fraction and trabecular thickness, more rod-like structure and the highest bone strength, and lateral specimens (lower right) had the lowest bone volume fraction and trabecular thickness, typical rod-like structure and relative higher bone strength.

Table I. Summary of the 3-D microstructural properties (mean and 95\% CI) of cancellous bone in the early osteoarthritis and in the control group

\begin{tabular}{|c|c|c|c|c|c|c|c|}
\hline Microstructural properties & Medial OA & Lateral intact & $\begin{array}{l}\mathrm{p} \text { value } \\
\left(\begin{array}{l}v \\
\mathrm{OAA}\end{array}\right)\end{array}$ & Normal medial & $\begin{array}{l}\mathrm{p} \text { value } \\
(v \text { OA })\end{array}$ & Normal lateral & $\begin{array}{l}\text { p value } \\
(v \text { LC })\end{array}$ \\
\hline (\%) & $5.1(22.1$ to 28.1$)$ & $0.2(16.8$ to 23.7$)$ & 0.0001 & $21.0(17.8$ to 24.3$)$ & 0.049 & 17.1 (14.7 to 19.5$)$ & 0.057 \\
\hline & 5) & & 005 & 5) & 047 & & 107 \\
\hline & .16 to 0.20$)$ & & 0004 & & & & 020 \\
\hline atio $\left(\mathrm{mm}^{-1}\right)$ & $15.1(1$ & 19.9) & 0.003 & 18.1 & 0.029 & 20.6 & 0.029 \\
\hline & & & & & & & 0.166 \\
\hline Connectivity density $\left(\mathrm{mm}^{-3}\right)$ & 6.84 (5.84 to 7.84$)$ & & 0.644 & & & $8.32(6.54$ to 10.1$)$ & 0.224 \\
\hline Mean trabecular volume $\left(\mu \mathrm{m}^{3}\right)$ & 39.8 (30.2 to 49.4$)$ & 29.7 (23.5 to 35.9$)$ & 0.005 & 28.5 (19.6 to 37.4$)$ & 0.067 & $21.9(17.2$ to 26.6$)$ & 0.035 \\
\hline Mer mo & 115.3 (99 to 131.6$)$ & $119.2(103$ to 135.4$)$ & 0.609 & $106.6(80.3$ to 133$)$ & 0.534 & $111.2(83.8$ to 139$)$ & 0.576 \\
\hline 3-D trabec & $0.58(0.54$ to 0.61$)$ & $0.60(0.56$ to 0.64$)$ & 0.260 & $0.588(0.52$ to 0.63$)$ & 0.754 & $0.59(0.53$ to 0.65$)$ & 0.929 \\
\hline Bone surface density $\left(\mathrm{mm}^{-1}\right)$ & $3.36(3.22$ to 3.50$)$ & $3.12(2.82$ to 3.41$)$ & 0.024 & 3.37 (3.06 to 3.69$)$ & 0.921 & 3.15 (2.82 to 3.48$)$ & 0.860 \\
\hline
\end{tabular}

The 3-D microstructural properties in the four groups are summarised in Table I.

Two-way analyses of variances showed that none of the condyles, by group interactions, was significant in analyses $(\mathrm{p}=0.537$ to 0.974$)$. Bone volume fraction was greater in the OA groups (both OA and lateral control) than in the normal groups (normal medial and lateral, $p=0.001$ for the group effect in the analysis of variance), and was greater 
Table II. Expression of degree of anisotropy (median, range) for cancellous bone specimens from normal tibiae and those with early OA. Because of the non-normal distribution of data, medians were presented

\begin{tabular}{|c|c|c|c|c|c|c|c|c|}
\hline $\begin{array}{l}\text { Degree of } \\
\text { anisotropy }\end{array}$ & Medial OA & $\begin{array}{l}95 \% \mathrm{CI} \\
\text { of mean }\end{array}$ & Lateral intact & $\begin{array}{l}95 \% \mathrm{CI} \\
\text { of mean }\end{array}$ & Normal medial & $\begin{array}{l}95 \% \mathrm{CI} \\
\text { of mean }\end{array}$ & Normal lateral & $\begin{array}{l}95 \% \mathrm{CI} \\
\text { of mean }\end{array}$ \\
\hline $\begin{array}{l}\text { Primary-to- } \\
\text { secondary }\end{array}$ & $2.92(1.05$ to 9.91$)$ & 2.17 to 3.67 & $3.12(1.05$ to 3.6$)$ & 2.55 to 3.69 & $3.65(1.14$ to 12.18$)$ & 2.61 to 4.69 & 3.81 (1.10 to 9.76$)$ & 3.05 to 4.57 \\
\hline $\begin{array}{l}\text { Secondary- } \\
\text { to-tertiary }\end{array}$ & 1.49 (1.08 to 2.70$)$ & 1.36 to 1.62 & 1.52 (1.05 to 3.06$)$ & 1.35 to 1.69 & 1.81 (1.06 to 9.59$)$ & 1.28 to 2.34 & 1.52 (1.06 to 3.58$)$ & 1.35 to 1.69 \\
\hline $\begin{array}{l}\text { Primary-to- } \\
\text { tertiary }\end{array}$ & $4.41(1.43$ to 14.81$)$ & 3.15 to 5.67 & $4.67(1.54$ to 11.96$)$ & 3.71 to 5.63 & $6.20(1.44$ to 23.39$)$ & 4.16 to 8.24 & 5.52 (1.64 to 16.4$)$ & 4.35 to 6.65 \\
\hline
\end{tabular}

medially than laterally ( $p=0.002$ for the effect of condyle in the analysis of variance). OA specimens had a $20 \%$ increase in bone volume fraction compared with that of the normal medial $(p=0.049$, Table I).

The structure model index was lower in the OA groups than in the normal groups $(\mathrm{p}=0.006)$, and was smaller medially than laterally $(\mathrm{p}=0.001)$. Medial OA specimens had a decrease of $25 \%$ in the structure model index compared with that of the normal medial (Table I).

Trabecular thickness was greater in the OA specimens than in the normal specimens $(p=0.002)$, and was greater medially than laterally $(\mathrm{p}=0.002)$. OA specimens had an increase of $12.5 \%$ in trabecular thickness compared with that of the normal medial, and the lateral intact samples had an increase of $23 \%$ in trabecular thickness relative to the normal lateral (Table I).

Bone-surface-to-volume ratio was lower in the OA specimens than in the normal specimens $(p=0.002)$, and was greater laterally than medially $(\mathrm{p}=0.030)$. OA specimens had a decrease of $16.6 \%$ in bone surface-to-volume ratio ( $p$ $=0.029$ ) compared with that of the normal medial, and the lateral control had a decrease of $13.1 \%$ in bone-surface-to- volume ratio relative to the normal lateral $(\mathrm{p}=0.029$, Table I).

Expression of the degree of anisotropy in three main directions in the OA and three controls are summarised in Table II. There were no significant differences between the groups. There does not appear to be transverse isotropy as evidenced by the fact that secondary-to-tertiary ratio differs significantly from 1 (Mann-Whitney rank sum test $\mathrm{p}=$ 0.001). All specimens in this study showed anisotropy, i.e. the ratios of three directions differ significantly from 1 (Mann-Whitney rank sum test $p=0.001$ ). All specimens in this study showed anisotropy, i.e., the ratios of three directions differ significantly from 1 (Mann-Whitney rank sum test $\mathrm{p}=0.001$ ).

The mean trabecular volume was greater in the OA specimens than in the normal specimens $(p=0.007)$, and was greater medially than laterally $(\mathrm{p}=0.018)$. The lateral control group had an increase of $35.6 \%$ relative to the normal lateral group (Table I).

Linear regression analysis showed that the ultimate stress of cancellous bone in OA was best explained by its linear relationship with the structure model index. The determina-

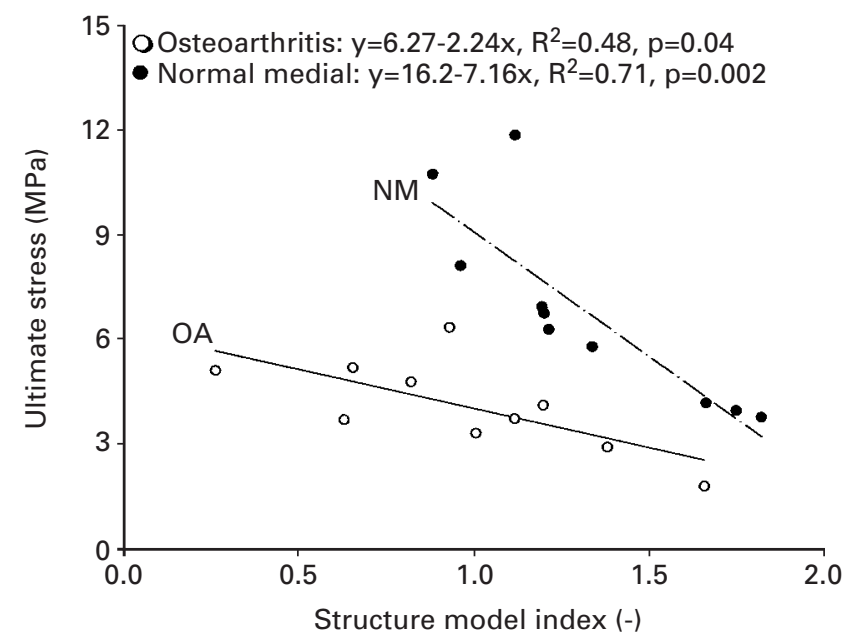

Fig. 2a

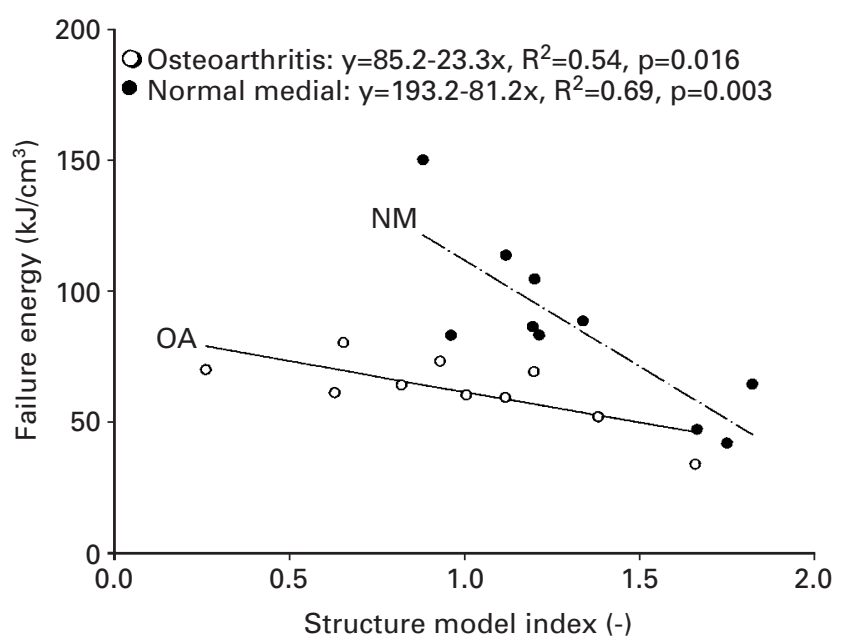

Fig. $2 b$

Linear regression analyses between a) ultimate stress or b) failure energy and the best predictors in the OA and normal medial groups. The slopes and intercepts of the linear regression lines in the OA group are significantly different from those in the normal medial $(\mathrm{p}=0.001)$. The determination coefficients $\left(\mathrm{r}^{2}\right)$ and $\mathrm{p}$ values are derived from linear regression. 
tion coefficient of ultimate stress in cancellous bone in OA was largely reduced compared with that of the normal medial (Fig. 2a); only $48 \%$ of the variance of ultimate stress could be explained. In normal medial controls, $71 \%$ of the variance of ultimate stress could be explained by the structure model index, and 54\% of the variance by the bonevolume fraction. More importantly, the slopes of two linear regression lines were significantly different $(p=0.001)$. For the OA group, the y-intercept (constant coefficient) of the linear model was significantly different from that of the normal medial ( $\mathrm{p}=0.001$, Fig. 2a).

Failure energy of cancellous bone in OA was also best explained by its linear relationship with the structure model index. The determination coefficient of failure energy in OA was significantly reduced compared with that in the normal medial control (Fig. 2b), and approximately 54\% variance of failure energy of cancellous bone in OA could be explained. In the normal medial control, $69 \%$ of the variance of failure energy could be explained by the structure model index alone. Similar to the associations between ultimate stress and structure model index, the slopes of two linear regression lines were significantly different $(\mathrm{p}=0.001)$. For the OA group, the $\mathrm{y}$-intercept (constant coefficient) of the linear model was significantly different from that of the normal medial group ( $p=0.001$, Fig. $2 b)$.

\section{Discussion}

Using unbiased 3-D methods, we have demonstrated microstructural changes in subchondral cancellous bone in human tibial early OA. Our data support the hypothesis that significant microstructural changes, in addition to changes of density, occur in subchondral cancellous bone in early OA. It was significantly thicker and unusually plate-like, but lower in mechanical properties than the normal bone. Similar to the OA group, microstructural changes for the control lateral condyle were also observed, with no sign of cartilage damage which may suggest an early alteration of the subchondral bone tissue. The structure model index best explained the mechanical properties of the four groups, but its ability to explain the mechanical properties of cancellous bone in OA was poor.

The cancellous bone structure shifts continuously from one type of structure to another during ageing, or bone remodelling. ${ }^{20,21}$ Previous investigations have shown that the structure differed between anatomical locations, and changed from more plate-like in younger cancellous bone towards more rod-like ageing cancellous bone. ${ }^{20,22}$ Cancellous bone was unusually plate-like in early OA even though the donors had a mean age of 74 years and a typical rod-like structure was observed for normal cancellous bone at this age. ${ }^{20}$ We have recently demonstrated that plate-like trabecular structure reflects high mechanical strength and rod-like trabecular structure reflects low mechanical strength. ${ }^{23}$ The change of structure from rods to plates in cancellous bone in early OA, however, does not enhance mechanical strength.
Cancellous bone in early OA had a greater bone-volume fraction and thicker trabeculae (increased by $20 \%$ and $12.5 \%$, respectively). An even greater increase by $51 \%$ in bone apparent density has been observed in late-stage OA. ${ }^{3}$

The orientation of trabeculae is important for the mechanical properties of cancellous bone. ${ }^{16,24}$ The bone density or structure model index together with the degree of anisotropy best predicts the mechanical properties of normal cancellous bone. ${ }^{23,25,26}$ It is of interest to note that cancellous bone in OA had a decrease in the degree of anisotropy and connectivity density, although it was statistically insignificant due to large variances in the data. The decrease in the degree of anisotropy is not uniformly distributed in all directions (Table II). The primary degree of anisotropy decreased by $37 \%$, much greater than the decreases in the other two degrees of anisotropy, i.e. the ratio between the eigen values of the secondary direction and the tertiary direction (decreased by about 20\%), and the ratio between the eigen values of the primary direction and the secondary direction (decreased by about $18 \%$ ). These results indicate that cancellous bone in OA is less well organised than normal cancellous bone, i.e. OA trabeculae orientated less well to the main loading direction of the tibia. This poor organisation may help to explain, at least in part, the significant decrease in the ability of cancellous bone in OA to carry mechanical load.

Our results of microstructural changes in subchondral cancellous bone in early OA suggest an interesting mechanism for bone remodelling. The increased trabecular thickness and bone density, but relatively decreased connectivity, indicate a process of filling trabecular remodelling cavities (inversion of perforation of trabecular plates ${ }^{27}$ ). This mechanism is followed by a progressive change of trabeculae from rods to plates in cancellous bone in early OA which is the opposite of the normal process of ageing in trabeculae which is normally a change from plates to rods. ${ }^{20}$ This process is also significantly different from the bone remodelling mechanism in normal ageing human tibial cancellous bone which may correct trabecular volume orientations, as clearly shown by a strong preferential alignment of trabeculae to the main loading direction and the constant nature of connectivity in ageing bone. ${ }^{28}$

Mean trabecular volume, mean marrow space volume, 3-D trabecular spacing, and bone surface density may provide additional information concerning the architecture of cancellous bone. None of these parameters has a great effect on the mechanical properties of cancellous bone when the structure model index or apparent density of bone is included in analyses.

In normal cancellous bone, the mechanical properties can be largely explained by bone density or structure model index. $^{16,23,26}$ This normal relationship, however, cannot simply be applied to cancellous bone in OA. Apart from the structure model index, none of the mechanical properties of cancellous bone in OA can be explained by the other measured microstructural properties. The amount of increase of 
bone tissue cannot entirely counteract for a total decrease of mechanical properties in early OA. The observed decrease of the ability to explain mechanical properties from densitymicrostructure may be interpreted as deterioration of the quality of bone in cancellous bone in OA. Therefore, the increased amount of defective bone tissue, which may also accompany the accumulation of microdamage, in both early $\mathrm{OA}$ and late OA could not entirely compensate the decrease in mechanical properties. 5,10

The mechanical strength of the medial condyle is significantly greater that that of the lateral condyle in normal tibiae. The axial compressive strength and stiffness distributions show similar patterns in normal human tibiae. ${ }^{29}$ OA mostly begins on the medial condyle of the tibia. In early OA, the changes predominantly occur on the central medial condyle resulting in the disruption of articular cartilage and the sclerosis of underlying bone, whereas the lateral condyle is virtually unaffected. This phenomenon may be explained by the strength distribution pattern on the tibial condyles, which is both seen in human tibial OA and an animal OA model. ${ }^{4,29,30}$ Only at the moderate or late stage, will changes occur on the lateral condyle. The strength distribution pattern may change in late $\mathrm{OA}^{31}$ Trabecular orientation in late OA was more vertical or perpendicular to the articular surface than that of the normal control, especially in the medial condylar periarticular subchondral cancellous bone. $^{32}$ The trabeculae of early OA were less anisotropic (i.e., less well aligned to the loading direction) than those of normal cancellous bone. These results may suggest an early disorganisation in the microstructure of cancellous bone in early OA with its architecture reorganising to adapt to mechanical loading as OA progresses.

It is of interest that the same trend was observed for the microstructural changes in the lateral samples from the tibiae with OA, when compared with those of the normal lateral, even though there was no sign of cartilage damage and the Mankin score was only 1.7. The cancellous bone at lateral intact condyle was also markedly plate-like, with thicker and fewer trabeculae, and was less anisotropic (Fig. 1, Table I), although there was no difference in the mechanical properties between both groups. While it is uncertain whether the lateral condyle would eventually develop to OA, it is a generally accepted concept that both medial and lateral condyles will develop OA in the later stages. This may suggest that an early change occurred in the subchondral bone tissue as a more global phenomenon in OA.

In the light of these data, our investigation has indicated a significant deterioration in the 3-D architecture of cancellous bone in early OA which leads to decreased mechanical properties. Our study provides further evidence that subchondral cancellous bone is involved and plays a major role in the pathogenesis of human tibial OA.

This study was supported by the Danish Rheumatism Association (Gigtforeningen, Grant no. 233-949-11.07.00 MP), Helga og Peter Kornings Fond and Hørslev-Fonden. We thank Anette Milton for technical assistance.

No benefits in any form have been received or will be received from a commercial party related directly or indirectly to the subject of this article.

\section{References}

1. Radin EL, Paul IL, Tolkoff MJ. Subchondral bone changes in patients with early degenerative joint disease. Arthritis Rheum 1970; 13:400-5.

2. Radin EL, Rose RM. Role of subchondral bone in the initiation and progression of cartilage damage. Clin Orthop 1986;213:34-40.

3. Burr DB, Schaffler MB. The involvement of subchondral mineralized tissues in osteoarthrosis: quantitative microscopic evidence. Microsc Res Tech 1997;37:343-57.

4. Bailey AJ, Mansell JP. Do subchondral bone changes exacerbate or precede articular cartilage destruction in osteoarthritis of the elderly? Gerontology 1997;43:296-304.

5. Li B, Aspden RM. Composition and mechanical properties of cancellous bone from the femoral head of patients with osteoporosis or osteoarthritis. J Bone Miner Res 1997;12:641-51.

6. Fazzalari NL, Parkinson IH. Fractal properties of subchondral cancellous bone in severe osteoarthritis of the hip. J Bone Miner Res 1997; 12:632-40.

7. Buckland-Wright JC, Lynch JA, Macfarlane DG. Fractal signature analysis measures cancellous bone organisation in macroradiographs of patients with knee osteoarthritis. Ann Rheum Dis 1996;55:749-55.

8. Dequeker J. Inverse relationship of interface between osteoporosis and osteoarthritis. J Rheumatol 1997;24:795-8.

9. Grynpas MD, Alpert B, Katz I, Lieberman I, Pritzker KP. Subchondral bone in osteoarthritis. Calcif Tissue Int 1991;49:20-6.

10. Fazzalari NL, Forwood MR, Smith K, Manthey BA, Herreen P. Assessment of cancellous bone quality in severe osteoarthrosis: bone mineral density, mechanics, and microdamage. Bone 1998;22:381-8.

11. Mankin HJ, Dorfman H, Lippiello L, Zarins A. Biochemical and metabolic abnormalities in articular cartilage from osteoarthritic human hips. II. Correlation of morphology with biochemical and metabolic data. J Bone Joint Surg [Am] 1971;53-A:523-37.

12. Ding M, Odgaard A, Hvid I. Accuracy of cancellous bone volume fraction measured by micro-CT scanning. J Biomech 1999;32:323-6.

13. Hildebrand T, Rüegsegger $P$. Quantification of bone microarchitecture with the structure model index. Comput Methods Biomech Biomed Engin 1997;1:15-23.

14. Hildebrand T, Rüegsegger P. A new method for the model-independent assessment of thickness in three-dimensional images. J Micro 1997; 185:67-75.

15. Odgaard A, Jensen EB, Gundersen HJ. Estimation of structural anisotropy based on volume orientation: a new concept. J Microsc 1990;157:149-62.

16. Goulet RW, Goldstein SA, Ciarelli MJ, et al. The relationship between the structural and orthogonal compressive properties of trabecular bone. J Biomech 1994;27:375-89.

17. Odgaard A, Gundersen HJ. Quantification of connectivity in cancellous bone, with special emphasis on 3-D reconstructions. Bone 1993; $14: 173-82$.

18. Odgaard A. Three-dimensional methods for quantification of cancellous bone architecture. Bone 1997;20:315-28.

19. Ding M, Dalstra M, Danielsen CC, et al. Age variations in the properties of human tibial trabecular bone. J Bone Joint Surg [Br] 1997;79B:995-1002.

20. Ding M, Hvid I. Quantification of age-related changes in the structure model type and trabecular thickness of human tibial cancellous bone. Bone 2000;26:291-5.

21. Kinney JH, Lane NE, Haupt DL. In vivo, three-dimensional microscopy of trabecular bone. J Bone Miner Res 1995;10:264-70.

22. Hildebrand T, Laib A, Muller R, Dequeker J, Ruegsegger P. Direct three-dimensional morphometric analysis of human cancellous bone: microstructural data from spine, femur, iliac crest, and calcaneus. J Bone Miner Res 1999;14:1167-74.

23. Ding M, Odgaard A, Danielsen CC, Hvid I. Mutual associations among microstructural, physical and mechanical properties of human cancellous bone. J Bone Joint Surg [Br] 2002;84-B:900-7.

24. Kabel J, van Rietbergen B, Odgaard A, Huiskes R. Constitutive relationships of fabric, density, and elastic properties in cancellous bone architecture. Bone 1999;25:481-6.

25. Ciarelli MJ, Goldstein SA, Kuhn JL, Cody DD, Brown MB. Evaluation of orthogonal mechanical properties and density of human trabecular bone from the major metaphyseal regions with materials testing and computed tomography. J Orthop Res 1991;9:674-82. 
26. Ulrich D, van Rietbergen B, Laib A, Ruegsegger $\mathbf{P}$. The ability of three-dimensional structural indices to reflect mechanical aspects of trabecular bone. Bone 1999;25:55-60.

27. Parfitt AM, Mathews CH, Villanueva AR, et al. Relationships between surface, volume, and thickness of iliac trabecular bone in aging and in osteoporosis: implications for the microanatomic and cellular mechanisms of bone loss. J Clin Invest 1983;72:1396-409.

28. Ding M, Odgaard A, Linde F, Hvid I. Age-related variations in the microstructure of human tibia cancelous bone. $J$ Orthop Res 2002;20:615-21.
29. Hvid I, Hansen SL. Trabecular bone strength patterns at the proximal tibial epiphysis. J Orthop Res 1985;3:464-72.

30. Matsui H, Shimizu M, Tsuji H. Cartilage and subchondral bone interaction in osteoarthrosis of human knee joint: a histological and histomorphometric study. Microsc Res Tech 1997;37:333-42.

31. Hvid I, Hansen SL. Subchondral bone strength in arthrosis: cadaver studies of tibial condyles. Acta Orthop Scand 1986;57:47-51.

32. Kamibayashi L, Wyss UP, Cooke TD, Zee B. Changes in mean trabecular orientation in the medial condyle of the proximal tibia in osteoarthritis. Calcif Tissue Int 1995;57:69-73. 